Annales Geophysicae (2003) 21: 577-581 (C) European Geosciences Union 2003

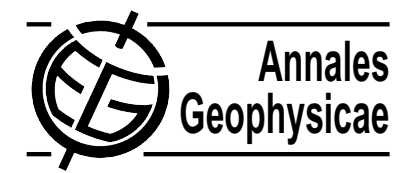

\title{
Mid-latitude ionospheric scintillation anomaly in the Far East
}

\author{
L. A. Hajkowicz ${ }^{1}$ and H. Minakoshi ${ }^{2}$ \\ ${ }^{1}$ Department of Physics, University of Queensland, Qld-4072, Australia \\ ${ }^{2}$ Communications Research Laboratories, Koganei-shi, Tokyo 184, Japan
}

Received: 12 April 2002 - Revised: 13 August 2002 - Accepted: 28 August 2002

\begin{abstract}
A long-term (over 3 years) study has been undertaken to obtain a comprehensive evaluation of VHF ionospheric scintillation morphology in East Asia (at Kokobunji in Japan), using amplitude records from Transit satellites. It is now evident that summer day and night scintillation enhancement in this mid-latitude region is a long-term evidence of a well-known Asian ionospheric disturbance anomaly. The scintillation activity is particularly strong during summer nights (21:00-24:00 LT) and on occasion, all satellite passes recorded on consecutive days are associated with pronounced scintillation activity. A second sub-maximum is observed in the summer pre-noon period (09:00-12:00 LT). The scintillation regions extend latitudinally for a distance of $400-600 \mathrm{~km}$ in the F-region and $100-200 \mathrm{~km}$ in the Eregion, mostly equatorwards of Kokobunji. For comparison similar scintillation data obtained for one year at the same longitudinal sector but in southern mid-latitudes (Brisbane in Australia) were compared with the simultaneous northern scintillation data. The scintillation activity at Brisbane was much less pronounced in the southern summer but was of the same low level during other seasons as that for Kokobunji. This consistent scintillation anomaly, as yet, has not been included in the global scintillation models, which are essential for radio-satellite communications.
\end{abstract}

Key words. Ionosphere (mid-latitude ionosphere; ionospheric irregularities)

\section{Introduction}

Global scintillation studies, over five decades, have been limited mainly to auroral and equatorial regions, as the intense ionospheric disturbances are usually limited to high and low latitudes. The empirical scintillation models clearly delineate these two latitudinal areas, with mid-latitudes being left over as insignificant for this type of study (Aarons, 1982). A num-

Correspondence to: L. A. Hajkowicz

(lech_hajkowicz@yahoo.co.uk) ber of southern mid-latitude scintillation studies indicate that on the whole, scintillation activity is low at these regions. The longest period of study of mid-latitude scintillations by Hajkowicz and Dearden (1988), spanning a solar cycle (1973-1985), indicates consistent diurnal, seasonal and solar cycle effects in scintillation magnitude and occurrences. More recently, Hajkowicz (1994) pointed out from a further five-year study (1987-1992) that localised mid-latitude scintillation patches occur most frequently at sunspot minimum, in association with the well-known occurrence maximum of mid-latitude spread-F. Hajkowicz (1989) studied scintillation occurrences from low mid-latitudes to auroral regions during sunspot maximum (1978-1981). A distinct seasonal pattern in scintillation activity was recorded in a wide range of southern mid-latitudes, with the principal maximum observed consistently during summer solstice. This trend departed from seasonal scintillation occurrences in the auroral regions, where maxima were observed during equinoctial periods.

Scintillation studies in East Asia (Japan),positioned in the same longitudinal sector as Australia, have been conducted using mainly VHF radio transmissions from geostationary satellites, limiting the detection range of scintillation activity to lower mid-latitudes. Sinno and Kan (1978 and 1980) used a geostationary satellite ETS-2 to observe VHF scintillations south of a mid-latitude station Kokobunji over a one year period (1977-1978). They observed summer scintillation maxima mainly at night but also during the day. Sinno and Minakoshi (1983) conducted a similar study in the same region and concluded that: "the occurrence of scintillation is much more extensive than indicated by previous investigations" (e.g. Aarons, 1982). Anomalous mid-latitude scintillations (exceeding $30 \mathrm{~dB}$ ) were observed in Japan from a geostationary satellite (Inmarsat) transmitting at a frequency of $1.5 \mathrm{GHz}$ (Karasawa et al.,1985). Fujita et al. (1978) used a geostationary ETS-II satellite to observe scintillations in the $1.7 \mathrm{GHz}$ range from Kokobunji over a short period. They observed strong nighttime and daytime scintillations, reaching maximum in June. Ogawa et al. (1989) used orbiting 


\section{TYPICAL SCINT. EVENTS-SUMMER}

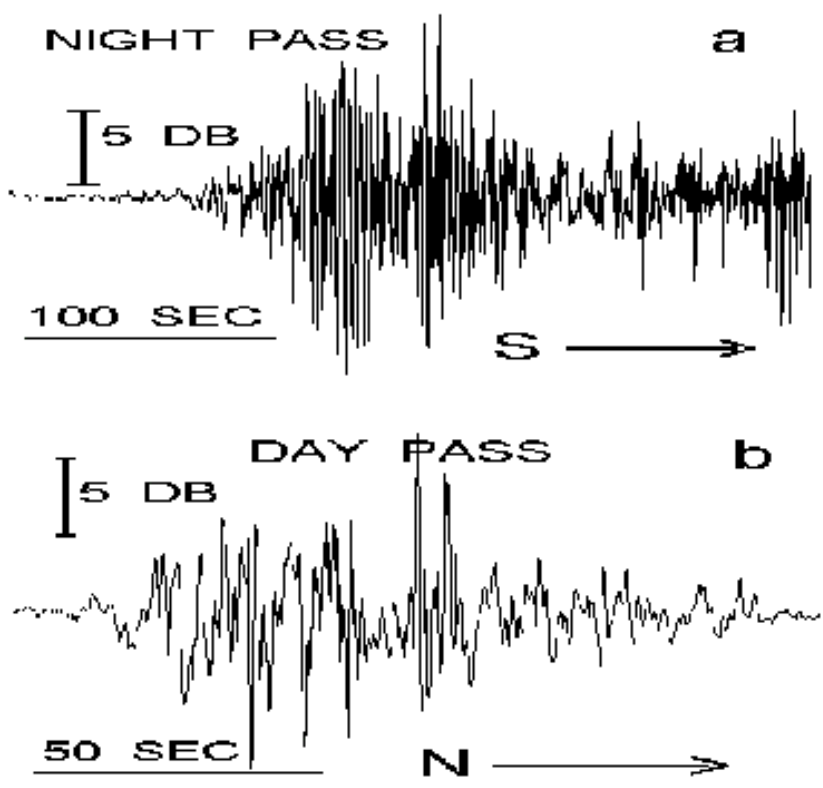

Fig. 1. Typical examples of enhanced summer scintillation activity in Far East during night (a) and day (b). The direction of the satellite travel is indicated (south-S or north-N).

Transit satellites in the summer of 1986 to observe scintillations at Kokobunji. They found a daytime maximum in scintillation occurrences coinciding with a well-known anomalous peak in the occurrence of sporadic-E over Japan in summer. Finally, Huang (1978) observed intense nighttime and daytime scintillations in summer in Lunping (Taiwan), some $20^{\circ}$. west of Japan. The latter result, although limited to a six-month study, indicates that the scintillation anomaly in East Asia has a considerable longitudinal extent.

It is evident that a cell of anomalous scintillation activity, limited to local summer, is located in mid-latitudes in East Asia. Although this has been known for some time, no sufficient stress was put on modifying the existing global scintillation models, largely ignoring mid-latitude scintillation activity which has a strong effect on radio-satellite communications and the positional fixes from the GPS satellites in this region. The aim of the present report is mainly to point out the consistent enhanced scintillation activity during summer for a period of more than three years.

\section{Method and results}

VHF radio-satellite transmissions (at a frequency of $150 \mathrm{Mhz}$ ), from polar orbit Transit satellites positioned at an altitude of $1000 \mathrm{~km}$, with an orbital period of $107 \mathrm{~min}$ (or $13.5 \mathrm{revs} /$ day), have been routinely recorded at a northern mid-latitude station, Kokobunji $\left(35.7^{\circ} \mathrm{N}\right.$ and $139.5^{\circ} \mathrm{E}$ geog., $25.6^{\circ} \mathrm{N}$ invar.lat) over 3 years (1998-2001) in the ascending part of the solar cycle. The recording was limited to satellite passes which were, at its nearest predicted approach time
Table 1. Sub-ionospheric horizontal extent of mid-latitude scintillations. Percentage occurrence is defined as the ratio of the number of passes with scintillations to the total number of passes recorded, expressed in percent

\begin{tabular}{ll}
\hline Length $(\mathrm{km})$ & Percentage occurrence $(\%)$ \\
\hline Nighttime (F-region) & \\
$<400 \mathrm{~km}$ & 10 \\
$400-600 \mathrm{~km}$ & 70 \\
$>600 \mathrm{~km}$ & 30 \\
Daytime (E-region) & \\
100-200 km & 70 \\
$200-300 \mathrm{~km}$ & 30 \\
\hline
\end{tabular}

(PTNA), within $30^{\circ}$ of zenith angle from the station. The geographic latitudinal ionospheric coverage for satellite passes was between 25 to $45^{\circ} \mathrm{N}$ (at the F-region, altitude $300 \mathrm{~km}$ ) and 30 to $40^{\circ} \mathrm{N}$ (at the E-region, altitude $100 \mathrm{~km}$ ). Almost 6000 satellite passes were recorded in a digital form (sampling rate of $70 \mathrm{~Hz}$ ). During the recording time, six Transit satellites (Oscar 23,25,27,29,31 and 32) were active.

Figure 1 shows typical structures of nighttime (a) and daytime (b) scintillation events recorded mainly in summer (May-July). The events were characterised by a relatively rapid onset followed by a well-defined maximum, with an extended tail end. Figure 2 shows the geographic location of the sub-ionospheric region where scintillations tended to occur, south of Kokobunji. The sub-ionospheric (for the Fregion at $300 \mathrm{~km}$ and E-region at $100 \mathrm{~km}$ ) latitudinal extents for which records were obtained are indicated. The horizontal extents of corresponding scintillations (from Fig. 1) are also shown. On the whole, $70 \%$ of the scintillations occurred south (equatorwards) of the station, whereas the reminder occurred north of the station, above $37^{\circ} \mathrm{N}$. Similar geographical distribution was found for daytime scintillation events. The horizontal extents of scintillations are summarised in Table 1. Scintillations used in this studies were limited to the events whose peak-to-peak amplitude was at least $2 \mathrm{~dB}$ within $10 \mathrm{~s}$ of recording.

It can be seen that the latitudinal width of scintillation regions was mainly between $400-600 \mathrm{~km}$ at night and between $100-200 \mathrm{~km}$ at daytime.

The prevalence of summer scintillation activity can be clearly seen from the histograms in Fig. 3. It can be seen that there is a sharp increase in the average scintillation magnitude (Fig. 3a) and occurrence number (Fig. 3b) during three summer months: May-July. The average scintillation magnitude represents the ratio of the sum of scintillation magnitudes to the total number of the passes in each month. The same definition applies to Figs. 4-6 for the specified time intervals. The narrow peak in year 2000 is due to a small number of available nighttime Transit passes in May and June; the majority of the close satellite passes were then during the day. The diurnal distributions of scintillation magnitude and 
MID-LAT. SCINT. REGION

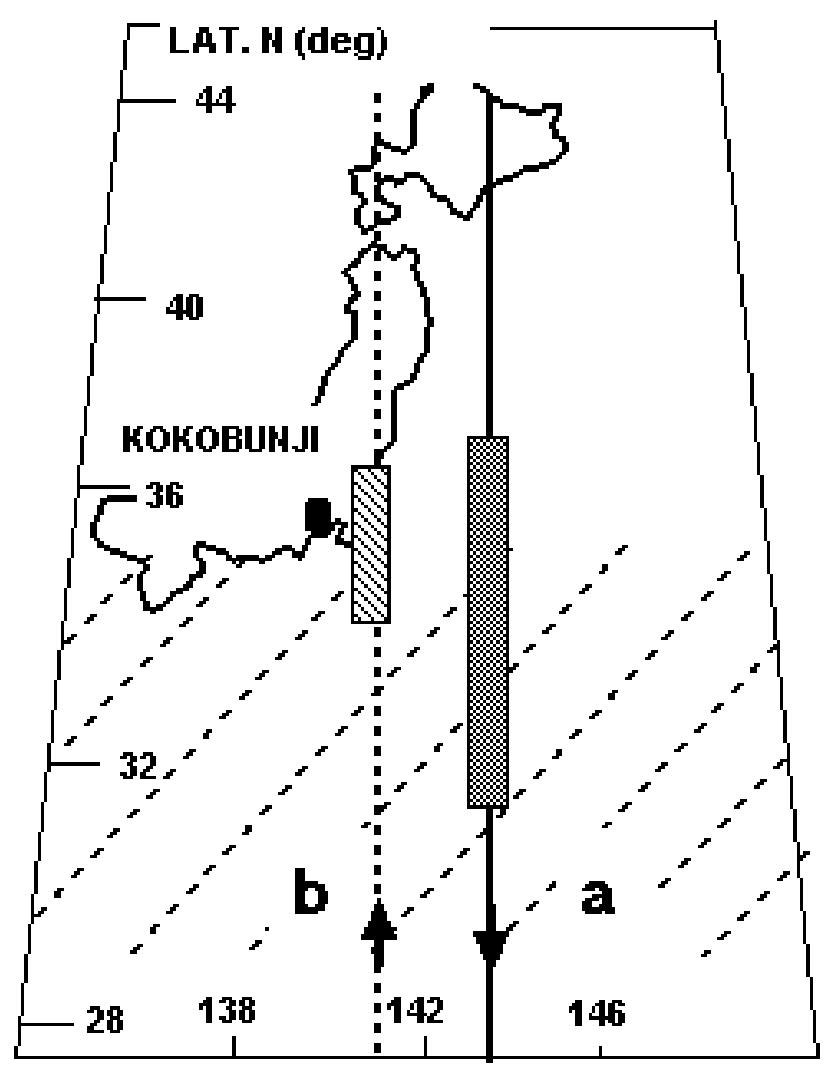

LONG.E(deg)

Fig. 2. The sub-ionospheric positions of scintillation regions as depicted in Fig. 1 during day (b, at the E-region) and night ( $a$, at the F-region). The shaded area refers to the most prevailing location of summer night scintillation activity, equatorwards of Kokobunji.

the percentage occurrence obtained for summer (as the occurrence in other seasons is negligible - Fig. 3) is shown in Fig. 4. It can be seen that the peak in the scintillation magnitude (averaging $7 \mathrm{~dB}$ ) and the percentage occurrence $(70 \%$ of all the recorded passes) were in the pre-midnight time interval (21:00-24:00 LT). It is of considerable interest that a peak in $\mathrm{GHz}$ scintillation activity was also observed by $\mathrm{Fu}-$ jita et al. (1978) at this time interval. A particularly intense period was in May 1998 when all the recorded passes in this time interval were associated with scintillations. A considerable smaller scintillation peak was observed before noon (09:00-12:00 LT).

The Transit recording during a period of July 1999-June 2000 was also conducted in another mid-latitude station (Brisbane, $27.5^{\circ} \mathrm{S}$ and $153^{\circ} \mathrm{E}$ geog., invar.lat. $36^{\circ} \mathrm{S}$ ), positioned in the same longitudinal sector as Kokobunji but in the Southern Hemisphere (Australia). A similar selection of passes as that for Kokobunji was made (i.e. primarily, the passes within $30^{\circ}$ of zenith angle were recorded). It is of considerable interest that the seasonal scintillation variations at Brisbane were much less pronounced both in magnitude

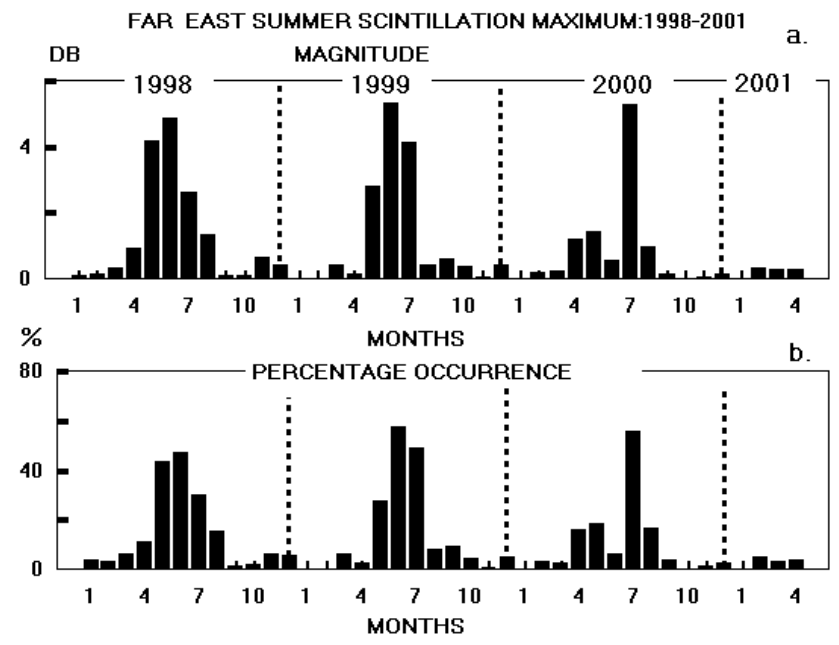

Fig. 3. The Far East summer scintillation anomaly in the average magnitude and the percentage occurrence number of scintillation events in the VHF range as recorded at Kokobunji over a three-year period.
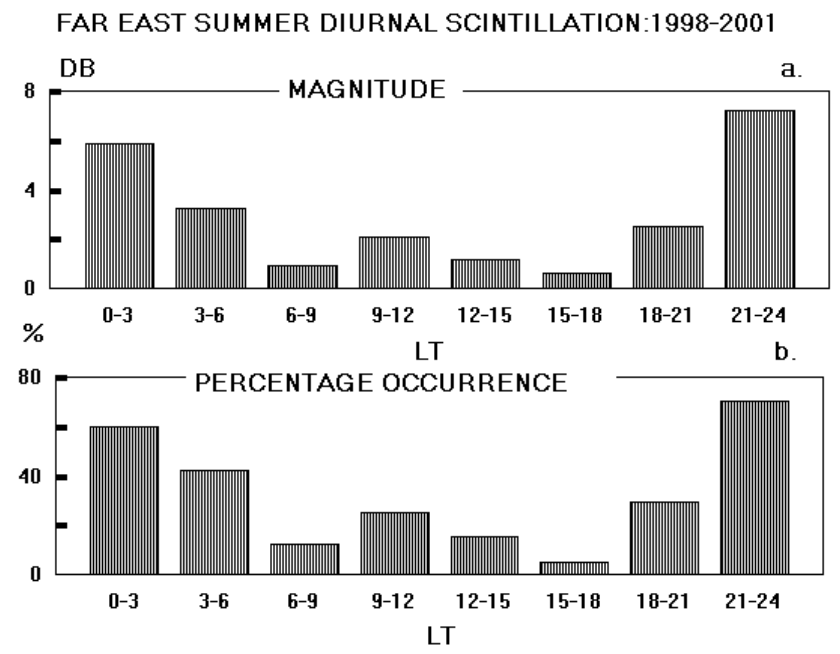

Fig. 4. The summer average diurnal scintillation magnitude and percentage occurrence of scintillation events at the indicated LT intervals as recorded at Kokobunji.

and occurrence number as compared to Kokobunji (Fig. 5). The single scintillation activity peak in January (southern summer) in Brisbane is much less outstanding than the scintillation activity in the northern summer months of JuneJuly. Figure 6 shows for comparison diurnal scintillation activity for two regions during local summer (May-July for Kokobunji and November-Jananuary for Brisbane) and for equinox (February-April and August-October). There was little scintillation activity for local winter for both stations (not shown). It is evident that summer scintillation activity in Japan at night and at the pre-noon period considerably exceeds the scintillation level in Australia (by a factor of 4 at 21:00-24:00 LT). The scintillation activity at both sites was similar at equinox (Fig. 6b). 

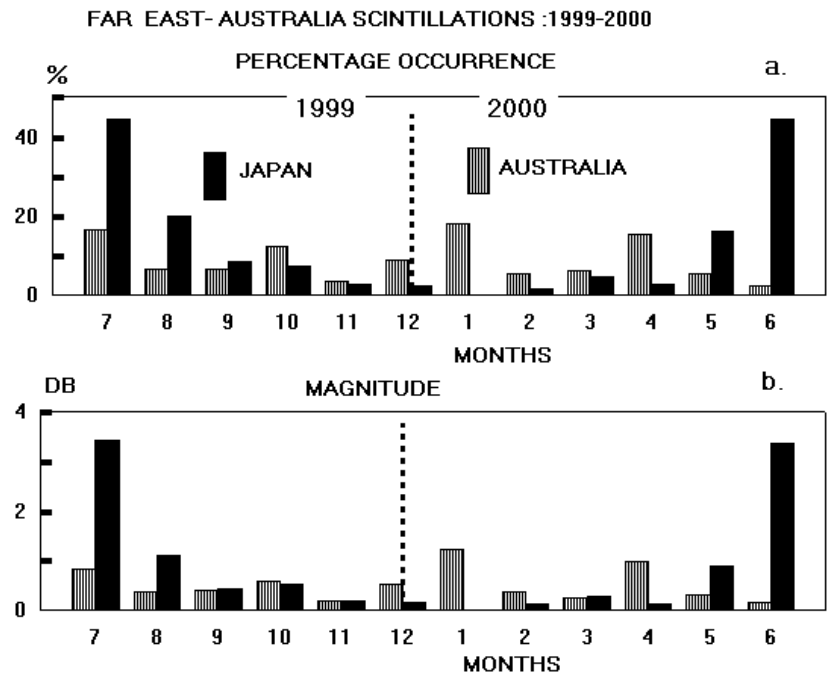

Fig. 5. Comparative mid-latitude scintillation occurrences (percentage occurrence and magnitude) in the Far East and Australia during one-year simultaneous recording.

\section{Conclusions and discussion}

The abnormally high scintillation activity in East Asia reported elsewhere was for the first time investigated over an extended period of time (3 years). In addition, one year of data during this period was available from a southern mid-latitude station. It became evident from the morphological study of scintillation activity that during northern summer, scintillations become much more pronounced than during other seasons. A particularly strong onset in scintillations takes place in the pre-midnight period (21:0024:00 LT) when, on occasion, almost all satellite passes are associated with strong scintillations. A smaller peak in daytime scintillations is observed in the pre-noon period (09:0012:00 LT). Scintillations tend to occur south (equatorwards) of the northern station, extending latitudinally $400-600 \mathrm{~km}$ in the F-region at night and $100-200 \mathrm{~km}$ in the E-region at day.

Simultaneous recording of scintillations at two midlatitude stations (Kokobunji in Japan and Brisbane in Australia) clearly indicate the anomalous scintillation enhancement in East Asia in northern summer. On the whole, scintillation activity is considerably smaller during local summer in southern mid-latitudes.

The unusual local increase in scintillations, which are associated with the generation of small-scale ionospheric irregularities, is yet another proof of the anomalous ionospheric disturbance levels occurring in summer in East Asia. Early studies of the global occurrence of sporadic-E by Smith and Finney (1960) pointed out that sporadic-E (Es) is three times more frequent in East Asia than in the North America in similar latitudes. They concluded that the abnormal ionospheric activity is due to the complexity of geomagnetic and geographic latitude (i.e. the magnetic equator is shifted polewards more than in other longitudinal sectors). Reddy and
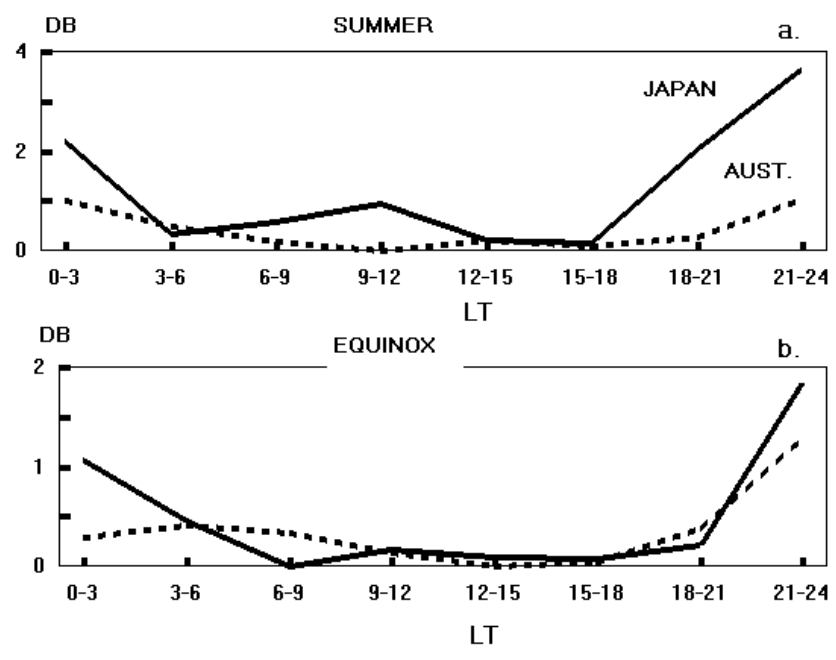

Fig. 6. Comparison of mid-latitude average diurnal scintillation levels in the Far East and Australia recorded during summer and equinox.

\section{EXAMPLES OF Es VARIATIONS IN JAPAN}
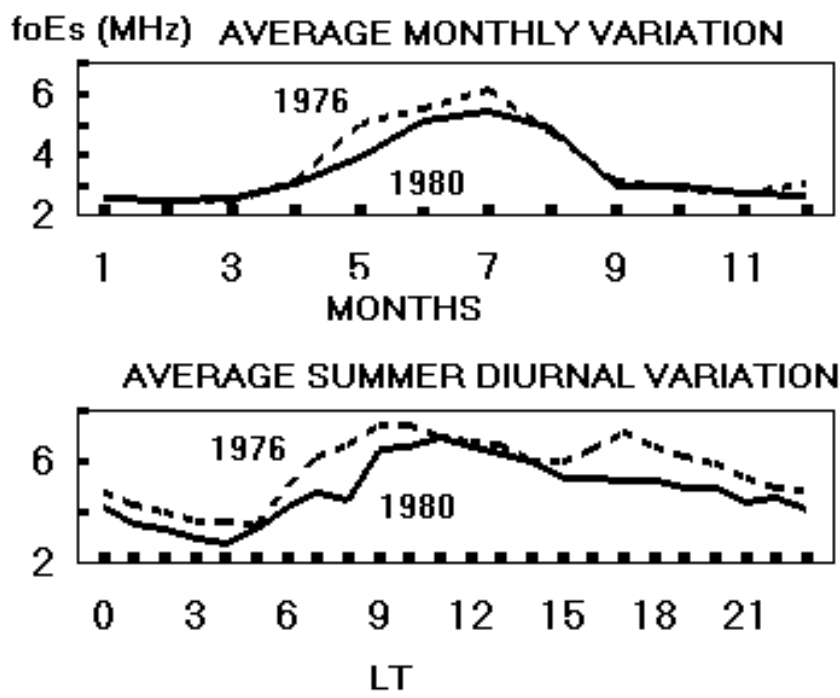

Fig. 7. Examples of average $f_{o}$ Es variations for two years at Kokobunji. The diurnal variation is shown for summer.

Matsushita (1969) studied in-depth blanketing Es in midlatitudes. They concluded that the occurrence of abnormally high $E s$ at Kokobunji (Japan) was purely a summer phenomenon, possibly associated with strong wind shear. The high level of scintillations in VHF/UHF and GHz range of radio satellite scintillations in summer in Japan, associated with the strong $E s$, has been reported consistently (c.f. the Introduction). Although no simultaneous records of the occurrence of $E s$ were available for this work, it is clear from other periods that large increases in the occurrence and intensity of $E s$ are present over Japan in summer. In particular, the yearly occurrence of Es was investigated for 1976 
and 1980 and it was found that the average intensity of the critical frequency of $E s$ ( $f o E s)$ had a distinct maximum in summer (Fig .7) The diurnal peak in the intensity of Es was in the late morning, coinciding with the peak of daytime scintillation activity, as shown in Fig. 4.

Sinno and Kan (1980) conducted scintillation studies over Japan from March 1977 to May 1978. They concluded that scintillations during summer nighttime are closely associated with the occurrence of spread-F, while those at daytime are due to sporadic-E. Huang (1978) reported a large increase in spread-F in East Asia (Taiwan) during summer months, contributing to night VHF scintillations. Since Taiwan is shifted longitudinally from the Japanese sector, it can be envisaged that the anomalous scintillation region forms a belt with considerable east-west horizontal extents. The sheet-like structures in southern mid-latitudes (Australia) scintillations were reported by Singleton (1970).

It follows from the present and previous results that the anomalous ionospheric activity creates a summer "hot spot" in the East-Asian mid-latitude ionosphere which is largely absent at other mid-latitudes. It concerns both the E- and F-region of the ionosphere. It seems that the F-region disturbances are particularly strong, as found from the present study. This anomaly has not received sufficiently wide attention in the global ionospheric scintillation models.

Acknowledgements. We are grateful for the financial support from Telstra, Australia and the Ministry of Telecommunications, Japan which made this project possible. The technical assistance from Mr. H. Lorek of the University of Queensland is greatly appreciated. We are also grateful to Mr. M. Grzesiak of the Space Research Centre, Warsaw, Poland for the computer software used in this project. We are also grateful to Dr. Ken Lynn for his helpful feedback on this report.

Topical Editor M. Lester thanks a referee for his help in evaluating this paper.

\section{References}

Aarons, J.: Global morphology of ionospheric scintillation, Proceedings of the IEEE, 70, 360-378, 1982.

Fujita, M., Ogawa, T., and Koike, K.: $1.7 \mathrm{GHz}$ scintillation measurements at midlatitudes using a geostationary satellite beacon, J. Atmos. Terr. Phys., 40, 963-968, 1978.

Hajkowicz, L. A.: Types of ionospheric scintillations in southern mid-latitudes during the last sunspot maximum, J. Atmos. Terr. Phys., 56, 391-399, 1994.

Hajkowicz, L. A.: Simultaneous recordings of VHF scintillation occurrences over a wide range of southern latitudes, Indian J Radio and Space Phys., 18, 2-9, 1989.

Hajkowicz, L. A. and Dearden, D. J.: Observations of random and quasi-periodic scintillations in southern mid-latitides over a solar cycle, J. Atmos. Terr. Phys., 50, 511-517, 1988.

Huang, Y. N.: Ionospheric scintillations at Lunping, J. Chinese Inst. Eng., 1, 81-84, 1978.

Karasawa, Y., Yasukawa, K., and Yamada, M.: Ionospheric scintillation measurements at $1.5 \mathrm{GHz}$ in mid-latitude region, Radio Sci., 20, 643-651, 1985.

Ogawa, T., Suzuki, A., and Kunitake, M.: Spatial distribution of mid-latitude sporadic-E scintillations in summer daytime, Radio Sci., 24, 527-538 1989.

Reddy, C. A. and Matsushita, S.: Time and latitude variations of blanketing sporadic-E of different intensities, J. Geophys. Res., 74, 824-843, 1969.

Singleton, D. G.: Dependence of satellite scintillations on zenith angle and azimuth, J. Atmos. Terr. Phys., 32, 789-803, 1970.

Sinno, K. and Minakoshi, H.: Experimental results on satellite scintillations due to field-aligned irregularities at mid-latitudes, J. Atmos. Terr. Phys., 45, 563-567, 1983.

Sinno, K. and Kan, M.: Mid-latitude ionospheric scintillations of VHF radio signals associated with peculiar fluctuations of Faraday rotation, J. Atmos. Terr. Phys., 40, 503-506, 1978.

Sinno, K. and Kan, M.: Ionospheric scintillation and fluctuation of Faraday rotation caused by spread-F and sporadic-E over Kokobunji, Japan, J. Radio. Res. Labs., 27, 53-77, 1980.

Smith, E. K. and Finney, J. W.: Irregularities of the ionosphere in the Far East. A report on IGY observations of sporadic-E and F-region scatter, J. Geophys. Res., 65, 885-892, 1960. 